Vol. 26, No. 02, pp. 88-98/Diciembre 2013

\title{
Producto Especial de convolución del Núcleo Ultrahiperbólico de Marcel Riesz.
}

\author{
Manuel A. Aguirre * and Emilio Aguirre R. \\ (Núcleo Consolidado de Matemática Pura y Aplicada(NuCOMPA) \\ Facultad de Ciencias Exactas Universidad Nacional del Centro \\ Pinto 399,(7000)Tandil, Argentina \\ e-mail: maguirre@exa.unicen.edu.ar
}

(Recibido/received: 19-Junio-2013; aceptado/accepted: 2130-Noviembre-2013)

\section{RESUMEN}

En este artículo primero consideramos la fórmula $R_{-2 k}(u)=\square^{k} \delta(x)$ para el caso especial $\mu$ par y $v$ y par y luego le damos sentido al producto de convolución $R_{-2 k}(u) * R_{-2 l}(u)$ para este caso, donde $R_{\alpha}(u)$ es el núcleo ultrahiperbólico definido por (8) y $\square^{k}$ es el operador definido por (16). Como consecuencia se obtiene la fórmula de $W_{-2 k}(u, m)$ y el producto de convolución de $\left\{\square+m^{2}\right\}^{k} \delta\{x\} *\left\{\square+m^{2}\right\}^{l} \delta\{x\}$, donde $W_{\alpha}(u, m)$ es la familia de funciones distribucionales definida (37) y $\left\{\square+m^{2}\right\}^{k}$ es definido por (39).

Palabras claves: Teoría de distribuciones; convolución de distribuciones.

Clasificación de AMS: 46F10,46F12.

\section{ABSTRACT}

In this article first let consider the formula $R_{-2 k}(u)=\square^{k} \delta(x)$ for the special case $\mu$ even and $v$ even and then we give a sense to the convolution product of $R_{-2 k}(u) * R_{-2 l}(u)$ for this case, where $R_{\alpha}(u)$ is the ultrahyperbolic kernel defined by (8) and $\square^{k}$ is the operator defined by (16). As consequence we obtain the formula of $W_{-2 k}(u, m)$ and the convolution product of $\left\{\square+m^{2}\right\}^{k} \delta\{x\} *\left\{\square+m^{2}\right\}^{l} \delta\{x\}$, where $W_{\alpha}(u, m)$ is the distributional family defined by (37) and $\left\{\square+m^{2}\right\}^{k}$ is defined by (39).

Keywords: Distributions Theory; convolution of distributions AMS Subject Classification: 46F10,46F12

\footnotetext{
"Work partially support by "Comisión de investigaciones Científicas (C.I.C.)" de la provincia de Buenos Aires, Argentina.
} 


\section{Introducción.}

Sea $x=\left(x_{1}, x_{2}, \ldots x_{n}\right)$ un punto de $R^{n}$ y sea

$$
x_{1}^{2}+\ldots+x_{\mu}^{2}-x_{\mu+1}^{2}-\ldots x_{\mu+\nu}^{2}=u,
$$

$\mu+v=n$ (dimensión del espacio). By $H_{+}$se designa el interior de un cono avanzado ó cono de avance:

$$
H_{+}=\left\{x \in R^{n}: x_{1}>0, u>0\right\}
$$

y por $\bar{H}_{+}$se designa su clausura. Similarmente, $H_{-}$designa el dominio

$$
H_{-}=\left\{x \in R^{n}: x_{1}<0, u>0\right\}
$$

y $\bar{H}_{-}$designa su clausura.

Sea $F(\lambda)$ una función de variable escalar $\lambda$, y sea $\Phi(x)$ una función dotada de las siguientes propiedades:

$$
\begin{aligned}
& \text { a) } \Phi(x)=F(u) \\
& \text { b) soporte } \Phi(x) \subset \bar{H}_{+} \\
& \text {c) } e^{\langle x, y\rangle} \Phi(x) \in L_{1} \quad \text { if } y \in V_{-},
\end{aligned}
$$

donde

$$
V_{-}=\left\{y \in R^{n}: y_{1}>0, y_{1}^{2}+\ldots+y_{\mu}^{2}-y_{\mu+1}^{2}-\ldots y_{\mu+v}^{2}>0\right\}
$$

Llamamos $R$ la familia de funciones $\Phi(x)$ que satisfacen las condiciones dadas en (4).

Similarmente, llamamos $A$ la familia de funciones que satisfacen las condiciones:

$$
\begin{aligned}
& \left.a^{\prime}\right) \Phi(x)=F(u) \\
& \left.b^{\prime}\right) \text { soporte } \Phi(x) \subset \bar{H}_{-} \\
& \left.c^{\prime}\right) e^{\langle x, y\rangle} \Phi(x) \in L_{1} \quad \text { if } y \in V_{+},
\end{aligned}
$$

donde

$$
V_{+}=\left\{y \in R^{n}: y_{1}<0, y_{1}^{2}+\ldots+y_{\mu}^{2}-y_{\mu+1}^{2}-\ldots y_{\mu+\nu}^{2}>0\right\}
$$


Vamos a considerar la siguiente familia de funciones $R_{\alpha}$ introducida por Y. Nozaki ([1], page 72):

$$
R_{\alpha}(u)=\left\{\begin{array}{lll}
\frac{u^{\frac{\alpha-n}{2}}}{K_{n}(\alpha)} & \text { si } & x \in H_{+} \\
0 & \text { si } & x \notin H_{+}
\end{array}\right\}
$$

Aquí $\alpha$ es un parámetro complejo y $n$ la dimensión del espacio.

la constante $K_{n}(\alpha)$, es definida por

$$
K_{n}(\alpha)=\frac{\pi^{\frac{n-1}{2}} \Gamma\left(\frac{2+\alpha-n}{2}\right) \Gamma\left(\frac{1-\alpha}{2}\right) \Gamma(\alpha)}{\Gamma\left(\frac{2+\alpha-\mu}{2}\right) \Gamma\left(\frac{\mu-\alpha}{2}\right)}
$$

donde, $\Gamma(\alpha)$ es la función gama, $\mu$ es el número de términos positivos de

$$
u=x_{1}^{2}+\ldots+x_{\mu}^{2}-x_{\mu+1}^{2}-\ldots x_{\mu+v}^{2}
$$

y $\mu+v=n$.

$R_{\alpha}(u)$, es una función ordinaria si $\operatorname{Re}(\alpha) \geq n$, y es una distribución de $\alpha$.

Llamaremos a $R_{\alpha}(u)$ el núcleo ultrahiperbólico de Marcel Riesz. Haciendo $\mu=1$ en (8) y (9) y usando la fórmula de duplicación de Legendre de $\Gamma(z)$

$$
\Gamma(2 z)=2^{2 z-1} \pi^{-\frac{1}{2}} \Gamma(z) \Gamma\left(z+\frac{1}{2}\right)
$$

([2], Vol. I, página 344, fórmula (15)), la fórmula (8) se reduce a

$$
M_{\alpha}(u)=\left\{\begin{array}{lll}
\frac{u^{\frac{\alpha-n}{2}}}{H_{n}(\alpha)} & \text { si } & x \in H_{+} \\
0 & \text { si } & x \notin H_{+} .
\end{array}\right\}
$$

Aquí

$$
u=x_{1}^{2}-x_{2}^{2}-\ldots x_{n}^{2}
$$

$\mathrm{y}$ 
Manuel A. Aguirre and Emilio Aguirre R.

$$
H_{n}(\alpha)=\pi^{\frac{n-2}{2}} 2^{\alpha-1} \Gamma\left(\frac{\alpha}{2}\right) \Gamma\left(\frac{\alpha-n+2}{2}\right) .
$$

$M_{\alpha}(u)$ es, precisamente, el núcleo hiperbólico de Marcel Riesz ([5], page 31).

Sea $R_{\alpha}(u)$ el núcleo ultrahiperbólico definido en (8), S.E. Trione en ([4], página 9, fórmula (III.9) demuestra la validez de la propiedad

$$
R_{-2 k}(u)=\square^{k} \delta(x)
$$

para $\mu$ impary $v$ par ( $n$ impar) y $k=0,1,2, \ldots$ donde

$$
\square^{k}=\left\{\frac{\partial^{2}}{\partial x_{1}^{2}}+\ldots \frac{\partial^{2}}{\partial x_{\mu}^{2}}-\frac{\partial^{2}}{\partial x_{\mu+1}^{2}}-\ldots \frac{\partial^{2}}{\partial x_{\mu+v}^{2}}\right\}^{k}
$$

es el operador ultrahiperbólico iterado $k$ veces y $\delta(x)=\delta\left(x_{1}, x_{2}, \ldots x_{n}\right)$ es la distribución delta de Dirac.

Los casos ( $\mu$ pary $v$ impar), ( $\mu$ par y $v$ par) y ( $\mu$ impary $v$ impar) han sido considerados en ([7]).

En particular se sabe de ([7], página 146, fórmula 2.53 y página147, fórmula 2.57) que la fórmula (15) es válida para los casos ( $\mu$ impary $v$ par) ( $n$ impar) y ( $\mu$ impar y $v$ impar) $(n$ par).

En este artículo primeramente vamos a considerar la fórmula (15) para el caso especial $\mu$ par y $v$ par y luego le daremos un sentido al producto de convolución de $R_{-2 k}(u) * R_{-2 l}(u)$ para este caso, donde $R_{\alpha}(u)$ es el núcleo ultrahiperbólico definido en (8). Como consecuencia se obtiene una fórmula para $W_{\alpha}(u, m)$ en $\alpha=-2 k . k=0,1,2, \ldots$ y se obtiene el producto de convolución $\left\{\square+m^{2}\right\}^{k} \delta\{x\} *\left\{\square+m^{2}\right\}^{l} \delta\{x\}$, donde $W_{\alpha}(u, m)$ es la familia de funciones distribucionales definda en (37).

Observemos que la constante definida en(form.9) usando la fórmula de duplicación de Legendre de $\Gamma(z)$ (ver fórmula (11)) se reduce a

$$
K_{n}(\alpha)=H_{n}(\alpha) \chi(\mu, \alpha)
$$

donde $H_{n}(\alpha)$ está definida en(14) y

$$
\chi(\mu, \alpha)=\frac{\Gamma\left(\frac{1-\alpha}{2}\right) \Gamma\left(\frac{1+\alpha}{2}\right)}{\Gamma\left(\frac{2+\alpha-\mu}{2}\right) \Gamma\left(\frac{\mu-\alpha}{2}\right)}
$$

luego la diferencia entre $K_{n}(\alpha)$ y $H_{n}(\alpha)$ es el factor $\chi(\mu, \alpha)$ definido en (18). 
Manuel A. Aguirre and Emilio Aguirre R.

Ahora usando las fórmulas

$$
\Gamma(z) \Gamma(1-z)=\frac{\pi}{\operatorname{sen}(z \pi)}
$$

y

$$
\Gamma\left(z+\frac{1}{2}\right) \Gamma\left(\frac{1}{2}-z\right)=\pi \sec (z \pi)
$$

De (18), se tiene

$$
\chi(\mu, \alpha)=\frac{\operatorname{sen}\left(\frac{\mu-\alpha}{2}\right) \pi}{\cos \left(\frac{\alpha}{2}\right) \pi}
$$

Por tanto de (21), se tiene

$$
\chi(\mu, \alpha)=(-1)^{\frac{\mu-1}{2}}
$$

si $\mu$ es impary

$$
\chi(\mu, \alpha)=\frac{(-1)(-1)^{\frac{\mu}{2}} \operatorname{sen}\left(\frac{\alpha}{2}\right) \pi}{\cos \left(\frac{\alpha}{2}\right) \pi}
$$

si $\mu$ es par.

De (17) y considerando (22) y (23) se tiene

$$
K_{n}(\alpha)=(-1)^{\frac{\mu-1}{2}} H_{n}(\alpha)
$$

si $\mu$ es impary

$$
K_{n}(\alpha)=\frac{(-1)(-1)^{\frac{\mu}{2}} \operatorname{sen}\left(\frac{\alpha}{2}\right) \pi}{\cos \left(\frac{\alpha}{2}\right) \pi} H_{n}(\alpha)
$$

si $\mu$ es par.

Por otra parte, de ([3],página 352), se tiene 


$$
\begin{gathered}
\operatorname{Re}_{\lambda=-\frac{n}{2}-k, k=0,1, \ldots} P_{+}^{\lambda}=\frac{(-1)^{\frac{n}{2}+k-1}}{\Gamma\left(\frac{n}{2}+k\right)} \delta_{1}^{\left(\frac{n}{2}+k-1\right)}+ \\
+\frac{(-1)^{\frac{v}{2}} \pi^{\frac{n}{2}}}{4^{k} k ! \Gamma\left(\frac{n}{2}+k\right)} \square^{k}\{\delta(x)\}
\end{gathered}
$$

si $\mu$ y $v$ son ambos pares ([3], página 352). Donde

$$
P_{+}^{\lambda}=\left\{\begin{array}{cc}
P^{\lambda} & \text { si } P \geq 0 \\
0 & \text { si } P \leq 0
\end{array}\right.
$$

([3], página 276), $P=P(x)=u(x)$ definida en (10) y $\square^{k}$ está definido en (16). En (26) $\delta_{1}^{(l)}(P)$ se entiende en el sentido de la reguralización de $\delta^{(l)}(P)\left([2]\right.$, page 249) si $l \geq \frac{n}{2}-1$.

\section{2. $R_{-2 k}(u)(\operatorname{caso} \mu y v$ par $)$}

Considerando que $u^{\frac{\alpha-n}{2}}$ tiene polos simple en los puntos $\alpha=-2 k, k=0,1, \ldots$ ([3], página 260) y tomando en cuenta que $\Gamma\left(\frac{\alpha}{2}\right)$ ([2], página, fórmula 8) tiene también polos simple en los mismos puntos, se tiene la siguiente fórmula,

$$
\begin{aligned}
& R_{-2 k}=\lim { }_{\alpha \rightarrow-2 k} R_{\alpha}(u)=\quad \quad \lim { }_{\alpha \rightarrow-2 k} \frac{\frac{u \frac{\alpha-n}{2}}{\Gamma\left(\frac{\alpha-n}{2}+1\right)}}{.} \\
& . \lim \frac{1}{\alpha \rightarrow-2 k} \frac{1}{2^{\alpha-1} \pi^{\frac{n-2}{2}} \Gamma\left(\frac{\alpha}{2}\right) \chi(\mu, \alpha)}=\quad \quad \lim \frac{\frac{u-n}{2}}{\Gamma \rightarrow-2 k} \Gamma^{\left.\frac{\alpha-n}{2}+1\right)} . \\
& \lim _{\alpha \rightarrow-2 k} \frac{\Gamma\left(1-\frac{\alpha}{2}\right) \cos \left(\frac{\alpha}{2}\right) \pi}{2^{\alpha-1} \pi \frac{n-2}{2} \pi(-1)(-1)^{\frac{\mu}{2}}}=\quad \frac{\operatorname{Res} u^{\frac{\alpha-n}{2}}}{\left.\operatorname{Res} \sum_{\alpha=-2 k}^{\frac{\alpha-n}{2}}+1\right)} . \\
& \lim _{\alpha \rightarrow-2 k} \frac{\Gamma\left(1-\frac{\alpha}{2}\right) \cos \left(\frac{\alpha}{2}\right) \pi}{2^{\alpha-1} \pi^{\frac{n-2}{2}} \pi(-1)(-1)^{\frac{\mu}{2}}}=\quad\left[\frac{k !(-1)^{k}}{2^{-2 k}(-1)(-1)^{\frac{\mu}{2}} \pi^{\frac{n}{2}}}\right] \\
& {\left[\delta_{1}^{\left(\frac{n}{2}+k-1\right)}(P)+\frac{(-1)^{\frac{v}{2}} \pi^{\frac{n}{2}}}{4^{k} k ! \Gamma\left(\frac{n}{2}+k\right)(-1)^{\frac{n}{2}+k-1}} \square^{k}\{\delta(x)\}\right] .}
\end{aligned}
$$


si $\mu$ y $\nu$ ambos son pares.

Ahora tomando en cuenta la siguiente fórmula

$$
\delta^{(s)}(u)=\frac{(-2)(-1)^{s}(-1)^{\frac{v}{2}} \pi^{\frac{n}{2}}}{4^{s-\frac{n}{2}+1}\left(s-\frac{n}{2}+1\right) !} \square^{s-\frac{n}{2}+1}\{\delta(x)\}
$$

si $\mu$ y $v$ son ambos pares y $s \geq \frac{n}{2}-1$ ([8], página 261, fórmula 54), donde $\square^{k}$ está definido en (16), se tiene la siguiente fórmula

Lema 1 La siguiente fórmula es válida

$$
R_{-2 k}(u)=(-1) \square \square^{k}\{\delta(x)\}
$$

si $\mu$ y $v$ son ambos pares. Donde $R_{\alpha}(u)$ es el núcleo ultrahiperbólico definido en (8).

Demostración: la fórmula (30) es consecuencia de las fórmulas (28) y (29), donde $\square^{k}$ está definido en (16).

Por otra parte, sabemos que la siguiente fórmula es válida,

$$
R_{-2 k}(u)=(-1) \square^{k}\{\delta(x)\}
$$

si $\mu$ y $v$ son ambo $\mu \mathrm{s}$ impares ([7],página 147, fórmula (2.57)).Por tanto de (30) y (31) se tiene la siguiente fórmula

$$
R_{-2 k}(u)=(-1) \square \square^{k}\{\delta(x)\}
$$

si $n$ es par.

Ahora de (32) y considerando la fórmula (15), se obtiene la siguiente fórmula

$$
R_{-2 k}(u)=(-1)^{n+1} \square^{k}\{\delta(x)\}
$$

bajo las condiciones:
a) $n \geq 2$
$y$
b) $0 \leq k$. 


\section{El producto de convolución de $R_{-2 k}(u) * R_{-2 l}(u)$}

Sabemos que la siguiente propiedad es válida

$$
R_{-2 k}(u) * R_{-2 l}(u)=R_{-2(k+l)}(u)
$$

si $n$ es impar ([12], página 123, fórmula (I,2,6)). Usando el producto de convolución

$$
\square^{t}\{\delta(x)\} * \square^{s}\{\delta(x)\}=\square^{t+s}\{\delta(x)\}
$$

si $n$ es par y $t, s$ son enteros no negativos ([9], página 346, fórmula (5.3)), se tiene la siguiente fórmula:

Lema 2 La siguiente fórmula es válida

$$
R_{-2 k}(u) * R_{-2 l}(u)=(-1)^{n+1} R_{-2(k+l)}(u)
$$

donde el símbolo * significa convolución.

Demostración: La fórmula (36) es consecuencia de las fórmulas (33), (34) y (35).

4. $W_{-2 k}(u, m)$

Consideremos la familia de funciones $R$ introducidas por S.E. Trione ([6])

$$
W_{\alpha}(u, m)=\left\{\begin{array}{cl}
\left(m^{-2} u\right)^{\frac{\alpha-n}{2}} J \frac{\alpha-n}{2}\left(\left(m^{2} u\right)^{\frac{1}{2}}\right) & \text { if } \mathrm{t} \in \Gamma_{+} \\
0 & \text { if } \mathrm{t} \notin \Gamma_{+}
\end{array}\right.
$$

donde $u$ está definida en (10), $\alpha$ es un parámetro complejo, $m$ es un número real no negativo, $n$ es la dimensión del espacio y $J_{\gamma}(z)$ designa la función de Bessel de primera clase definida por la fórmula:

$$
J_{\gamma}(z)=\sum_{p=o}^{\infty} \frac{(-1)^{p}\left(\frac{x}{2}\right)^{\gamma+2 p}}{p ! \Gamma(\gamma+p+1)} .
$$

$W_{\alpha}(u, m)$ la cual es una función ordinaria si $\operatorname{Re}(\alpha) \geq n$, es una distribución de $\alpha$. 
Escribimos por definición

$$
\left\{\square+m^{2}\right\}^{k}=\left\{\frac{\partial^{2}}{\partial x_{1}^{2}}+\ldots \frac{\partial^{2}}{\partial x_{\mu}^{2}}-\frac{\partial^{2}}{\partial x_{\mu+1}^{2}}-\ldots \frac{\partial^{2}}{\partial x_{\mu+v}^{2}}+m^{2}\right\}^{k}
$$

donde $\mu+v=n$ es la dimensión del espacio y $m$ un número real, $\left\{\square+m^{2}\right\}^{k}$ es el operador $n-$ dimensional ultrahiperbólico de Klein Gordon iterado $k$ veces.

De ([6]) se tiene la siguiente fórmula:

$$
W_{\alpha}(u, m)=\sum_{p=o}^{\infty}\left(\begin{array}{c}
-\frac{\alpha}{2} \\
p
\end{array}\right)\left(m^{2}\right)^{p} R_{\alpha+2 p}(u)
$$

donde $R_{\lambda}(u)$ es la familia de funciones distribucionales introducida por Nozaki ([1], página 72) definida por la fórmula (9) $\mathrm{y}$

$$
\left(\begin{array}{c}
-\frac{\alpha}{2} \\
p
\end{array}\right)=\frac{\Gamma\left(1-\frac{\alpha}{2}\right)}{p ! \Gamma\left(1-\frac{\alpha}{2}-p\right)}
$$

Haciendo $\alpha=-2 k, k=0,1, \ldots$ en (41) y usando (19) se tiene

$$
\left(\begin{array}{c}
-\frac{\alpha}{2} \\
p
\end{array}\right)=\left\{\begin{array}{ccc}
\left(\begin{array}{l}
k \\
p
\end{array}\right) & \text { if } & 0 \leq p \leq k \\
0 & \text { if } & p>k .
\end{array}\right.
$$

De (40), usando (33) y (42) se tiene,

$$
W_{-2 k}(u, m)=\sum_{p=o}^{k}\left(\begin{array}{c}
k \\
p
\end{array}\right)\left(m^{2}\right)^{p} R_{-2(k-p)}(u)=\sum_{p=o}^{k}\left(\begin{array}{l}
k \\
p
\end{array}\right)\left(m^{2}\right)^{p} \square^{k-p}\{\delta(x)\}
$$

Por otra parte, considerando la fórmula

$$
\left\{\square+m^{2}\right\}^{-\frac{\alpha}{2}}=\sum_{p=o}^{\infty}\left(\begin{array}{c}
-\frac{\alpha}{2} \\
p
\end{array}\right)\left(m^{2}\right)^{p} \square^{-\frac{\alpha}{2}-p}
$$

dada por S.E. Trione in ([6]) y tomando en cuenta la propiedad (42) se tiene 
Manuel A. Aguirre and Emilio Aguirre R.

$$
\left\{\square+m^{2}\right\}^{k}\{\delta(x)\}=\sum_{p=o}^{k}\left(\begin{array}{c}
k \\
p
\end{array}\right)\left(m^{2}\right)^{p} \square^{k-p}\{\delta(x)\}
$$

De (40), (43) y (45) se tiene la siguiente fórmula

$$
W_{-2 k}(u, m)=(-1)^{n+1}\left\{\square+m^{2}\right\}^{k}\{\delta(x)\}
$$

donde $n$ es la dimensión del espacio.

La fórmula

$$
W_{-2 k}(u, m)=\left\{\square+m^{2}\right\}^{k}\{\delta(x)\}
$$

fue probada por S.E. Trione en ([6]).

Podemos observar que las fórmulas (46) y (47) son iguales para el caso $n$ impar.

Usando las fórmulas (46), (44) y (36) y tomando en cuenta la fórmula

$$
\left(\sum_{s=o}^{t}\left(\begin{array}{l}
k \\
s
\end{array}\right)\left(\begin{array}{c}
l \\
t-s
\end{array}\right)\right)=\left(\begin{array}{c}
k+l \\
t
\end{array}\right)
$$

se tiene

$$
\begin{gathered}
(-1)^{n+1}\left\{\square+m^{2}\right\}^{k}\{\delta(x)\} *(-1)^{n+1}\left\{\square+m^{2}\right\}^{l}\{\delta(x)\}= \\
=W_{-2 k}(u, m) * W_{-2 l}(u, m)= \\
=\sum_{p=o}^{\infty}\left(\begin{array}{c}
k \\
p
\end{array}\right)\left(m^{2}\right)^{p} R_{-2(k-p)}(u) * \sum_{j=o}^{\infty}\left(\begin{array}{l}
l \\
j
\end{array}\right)\left(m^{2}\right)^{j} R_{-2(l-j)}(u)= \\
=\sum_{t=o}^{\infty}\left(\sum_{s=o}^{t}\left(\begin{array}{c}
k \\
j
\end{array}\right)\left(\begin{array}{c}
l \\
t-j
\end{array}\right)\right)\left(m^{2}\right)^{t}\left[R_{-2(k-p)}(u) * R_{-2(l-j)}(u)\right]= \\
\sum_{t=o}^{\infty}\left(\begin{array}{c}
k+l \\
t
\end{array}\right)\left(m^{2}\right)^{t}(-1)^{n+1} R_{-2(k+l-t)}(u)= \\
=(-1)^{n+1} W_{-2(k+l)}(u, m)=\left\{\square+m^{2}\right\}^{k+l}\{\delta(x)\} .
\end{gathered}
$$

De (49) se tiene la siguiente fórmula:

Vol. 26, No. 02, pp. 88-98/Diciembre 2013 
Lema 3 La siguiente fórmula es válida

$$
\left\{\square+m^{2}\right\}^{k} \delta(x) *\left\{\square+m^{2}\right\}^{l}\{\delta(x)\}=(-1)^{n+1}\left\{\square+m^{2}\right\}^{k+l}\{\delta(x)\}
$$

donde $n$ es la dimensión del espacio.

Demostración: La fórmula (50) es consecuencia de la fórmula (49).

La fórmula (50) es una generalización de la fórmula

$$
\{\square\}^{k} \delta(x) *\{\square\}^{l}\{\delta(x)\}=\{\square\}^{k+l}\{\delta(x)\}
$$

la cual aparece en ([9], página 346, fórmula (5.3)).

\section{Referencia}

[1] Y. Nozaki, On Riemann-Liouville integral of ultrahyperbolic type, Kodai Mathematical Seminar Reports, 6 (2):6987(1964).

[2] A. Erdelyi, Ed. Higher Trascendental Functions, Vol.I and II, McGraw-Hill, New York, 1953.

[3] I.M.Gelfand and G.E.Shilov., Generalized Functions, Vol.I, Academic Press, New York, 1964.

[4] Trione S.E., On Marcel Riesz'Ultrahyperbolic Kernel, prepint 116, Trabajos de Matemática, Instituto Argentina de Matemática-CONICET,1987.

[5] Riesz M., L 'integrale de Riemann-Liouville et le problème de Cauchy, Acta Math. 81,1-223,1949.

[6] Trione S.E., On the elementary retarded, ultrahyperbolic solution of the Klein-Gordon operator, iterated k-times, Studies in Applied Mathematics, Massachusetts Institute of Thecnology,Cambridge,Massachuesetts,U.S.A., 79,pp.121141,1988 .

[7] Aguirre M. A., The Distributional Hankel Transform of Marcel Riesz's Ultrahyperbolic Kernel,Studies in Applied Mathematics 93:133-162,1994

[8] Aguirre M. A., Proportionality of k-th derivative of Dirac delta in the hypercone, Mathematica Balkanica, New Series Vol.14, 2000, Fasc.3-4.

[9] Aguirre M. A., The distribution $\delta^{(k)}\left(P \pm i o-m^{2}\right)$, Journal of Computational and Applied Mathematics, 88 (1977), 339-348.

[10] Aguirre M. A., The convolution product of $W_{\alpha}(u, m) * W_{\beta}(u, m)$, Mathematicae Notae, Vol.38, 1995/96, Rosario, República Argentina.

[11] Aguirre M. A., New formulae about the residue of distributions $P_{ \pm}^{\gamma}$ (Nuevas fórmulas acerca del residuo de la distribución $P_{ \pm}^{\gamma}$, NEXO (Revista Científica) vol.20,No. 01.pp.35-45,2007.

[12] Aguirre M. A.and Trione S.E.,The distributional convolution products of Marcel Riesz 'ultra-hyperbolic kernel, Revista de la Unión Matemática Argentina, Volumen 39, 1995.

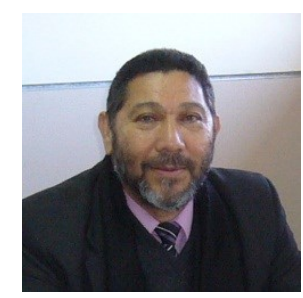

\section{Manuel A. Aguirre, Profesor y Decano}

Facultad de Ciencias Exactas

Universidad Nacional del Centro de la Prov. de Buenos Aires

Paraje Arroyo Seco, 7000-Tandil

Provincia de Buenos Aires, Argentina

Tel.: +54 2293439657

E-mail: maguirre@exa.unicen.edu.ar 